Artículo

\title{
Estimación del factor de transporte del índice de fósforo con climatologías y escenarios de cambio climático en tierras de Jalisco, México
}

Hugo Ernesto Flores López ${ }^{\text {a }}$

Álvaro Agustín Chávez Durán a

José Ariel Ruíz Corral ${ }^{\mathrm{b}}$

Celia De La Mora Orozco ${ }^{a^{*}}$

Uriel Figueroa Viramontes ${ }^{c}$

Agustín Hernández Anaya d

a Instituto Nacional de Investigaciones Forestales, Agrícolas y Pecuarias (INIFAP). Campo Experimental Centro-Altos de Jalisco. Tel: 01800 0882222. AV. Biodiversidad 2470. 47600 Tepatitlán de Morelos. Jalisco, México.

${ }^{\mathrm{b}}$ Universidad de Guadalajara. Centro Universitario de Ciencias Biológicas y Agropecuarias. Camino Ramón Padilla Sánchez No. 2100 Nextipac, 44600, Zapopan, Jalisco, México.

${ }^{\mathrm{c}}$ INIFAP. Campo Experimental La Laguna. Coahuila. México.

${ }^{\mathrm{d}}$ Universidad de Guadalajara. Centro Universitario de los Altos. México.

* Autor de correspondencia: delamora.celia@inifap.gob.mx

\section{Resumen:}

El índice de fósforo (IP) es una herramienta de planeación para identificar los campos agrícolas o ganaderos con potencial de aporte de fósforo para los cuerpos de agua y distinguir prácticas de manejo de nutrientes que favorecen este proceso. El factor de transporte del IP (FTIP), tiene implícitos elementos no controlables del ambiente, como la lluvia, la cual da incertidumbre a la agricultura y es favorecido por el proceso de cambio climático actual. En México pocos estudios se han realizado con el FTIP, por lo que el objetivo del presente trabajo fue aplicar la metodología de cálculo para el FTIP e identificar áreas vulnerables a la 
pérdida de fósforo de las tierras a los cuerpos de agua en dos escenarios de cambio climático y tres climatologías de Jalisco. Se utilizó el modelo de IP de Gburek, aplicado en dos rutas representativas de concentración de gases efecto invernadero (RCP 4.5 y 8.5), con las climatologías 2030, 2050 y 2070, y 2010 como línea base. En el cálculo del FTIP se utilizaron ARCGIS y GIS IDRISI. Los resultados mostraron niveles de vulnerabilidad a la pérdida de fósforo de muy bajo a alto en la línea base, mientras en el RCP4.5 se calificó el FTIP como muy bajo a medio y en la RCP8.5 fue de muy bajo a alto. Un elemento que resultó sobresaliente en el FTIP fue la alta vulnerabilidad de los predios con poca distancia a la red de drenaje o cuerpo de agua.

Palabras clave: Pérdida de fósforo, Riesgo ambiental, Calidad de agua.

Recibido: 20/11/2017

Aceptado: 22/08/2018

\section{Introducción}

La pérdida de fósforo $(\mathrm{P})$ de fuentes de contaminación difusas agrícolas y ganaderas, son las causas principales de eutrofización de agua dulce en las regiones agrícolas en los países desarrollados $^{(1,2)}$ y en países en desarrollo como México ${ }^{(3,4)}$. En algunas regiones de México, con elevada concentración de ganado, como los Altos de Jalisco ${ }^{(4)}$, o con alta intensidad de uso de la tierra, como la región centro de Jalisco ${ }^{(5)}$, los efectos son visibles en los cuerpos de agua superficial por el crecimiento desmedido de algas y malezas acuática ${ }^{(6-10)}$.

Una manera como se ha abordado esta problemática, es mediante el uso del Índice de Fósforo $(\mathrm{IP})^{(11)}$. En Estados Unidos de Norteamérica se utiliza como herramienta común para la planeación estratégica del uso de nutrientes ${ }^{(12)}$. El IP permite identificar el potencial de aporte de $\mathrm{P}$ por los campos agrícolas o ganaderos hacia los cuerpos de agua y distinguir las prácticas de manejo que reducen las pérdidas de $\mathrm{P}$ y que contribuyen a la conservación de la calidad del suelo y el agua ${ }^{(13)}$. El IP se ha evaluado y calibrado para los Altos de Jalisco ${ }^{(9)}$.

El IP se caracteriza por tener dos tipos de factores: 1) los factores de transporte de P que se constituyen por la erosión del suelo, el escurrimiento superficial y la distancia del predio a una red de drenaje o cuerpo de agua superficial (conectividad) y 2) la fuente de $\mathrm{P}$, formada por el contenido de fósforo en el suelo, la frecuencia y método de aplicación de fertilizantes químicos y fuentes orgánicas de $\mathrm{P}^{(11)}$. Los factores de transporte en el Índice de P (FTIP) consideran la transferencia del $\mathrm{P}$ disuelto en el escurrimiento y $\mathrm{P}$ adsorbido en sedimentos, los cuales viajan del predio a los cuerpos de agua superficiales o red de drenaje superficial. 
El FTIP tiene implícitos elementos no controlables del ambiente, como la lluvia, el cual da a la agricultura alta incertidumbre para la producción, pero también a los mecanismos de transporte del P. Cada factor se clasifica en cinco niveles de vulnerabilidad de acuerdo con la intensidad que ocurre cada uno, calificación que posteriormente se multiplica por un valor de ponderación. El FTIP resulta de la multiplicación de cada factor ponderado para obtener niveles de vulnerabilidad a la pérdida de $\mathrm{P}$ con valores de 0 a 1 . Finalmente el valor del FTIP se multiplica por el factor fuente de $\mathrm{P}$ para obtener el $\mathrm{IP}^{(14,15)}$.

Desde un punto de vista de cambio climático, se prevé una variedad de efectos sobre los ecosistemas asociados con procesos climatológicos e hidrológicos con eventos extremos relacionadas a inundaciones, grandes escurrimientos de agua, períodos de sequía o sequías, e incendios forestales, con implicaciones directas sobre el FTIP ${ }^{(16,17,18)}$. Pero son de particular importancia los efectos de eventos extremos de lluvia que provocan erosión de suelo y causan una grave degradación de la tierra y deterioro ambiental ${ }^{(1,19,20)}$. La Ecuación Universal de Pérdida de Suelo (USLE, por sus siglas en ingles), es utilizada para estimar la erosión hídrica en el $\mathrm{IP}^{(9)}$. El factor de erosividad de la lluvia (R) de la USLE, determina la fuerza potencial actual de la erosión por lluvia ${ }^{(19,21)}$, como los efectos esperados en el futuro con el cambio climático $^{(22)}$.

Por las razones anteriores, el cambio en los patrones de lluvia y el escurrimiento superficial procedente de la modificación del clima, han generado una fuerte incertidumbre en la agricultura y ganadería de Jalisco, particularmente por la posibilidad del aumento en la contaminación difusa que estas actividades primarias generan. De ahí la necesidad de evaluar el FTIP, bajo climatologías y escenarios de cambio climático de tierras en Jalisco. El objetivo del presente trabajo fue aplicar la metodología de cálculo para el factor de transporte del Índice de Fósforo e identificar áreas vulnerables a la pérdida de fósforo de las tierras a los cuerpos de agua en dos escenarios de cambio climático y tres climatologías de Jalisco, México.

\section{Material y métodos}

El estudio se desarrolló para el estado de Jalisco, México. Tiene una superficie de 1'487,832 ha, con $3.26 \%$ de uso forestal, $64.82 \%$ tiene uso pecuario, $21.84 \%$ uso agrícola y $10.08 \%$ con uso diferente. De la superficie agrícola, 292,903 ha se siembran con riego y 1'343,167 ha de temporal ${ }^{(23)}$. La mayor proporción de la superficie con uso pecuario, es un indicador de la importancia que tiene esta actividad en Jalisco, en la cual la agricultura representa un soporte importante a este sector y como actividad primaria, donde el cultivo de maíz ocupa $72.1 \%$ de la superficie. 
La precipitación promedio anual de Jalisco en el periodo de 1961 a 2010 fue de 897 mm, con un máximo de 1,934 mm y un mínimo $461 \mathrm{~mm}$. Al respecto, el $82.9 \%$ de la lluvia se concentra en los meses de junio a septiembre, con la mayor cantidad en julio.

Se utilizaron los componentes del Factor de Transporte Índice de Fósforo (FTIP) de Gburek et al. $^{(11)}$. El valor del FTIP obtenido varía de 0 a 1 , con nivel de vulnerabilidad muy bajo cuando FTIP es menor a 0.15 , bajo de 0.15 a 0.3 , medio de 0.3 a 0.5 , alto de 0.5 a 0.8 y muy alto mayor que $0.8^{(24)}$.

\section{Estimación de los Factores de Transporte del Índice de Fósforo (FTIP)}

El proceso de evaluación de los FTIP se resumió en el modelo conceptual mostrado en la Figura 1, para obtener los niveles de vulnerabilidad a la pérdida de fósforo. Esta figura describe el proceso de estimación de la erosión hídrica con la USLE constituida por los factores erosividad de la lluvia $(\mathrm{R})$, erosionabilidad del suelo $(\mathrm{K})$, los factores longitud e inclinación de la pendiente (LS), la cobertura del suelo (C) y las prácticas de manejo (P); el escurrimiento superficial anual se evalúa con el Número de Curva y la Distancia de contribución de fósforo por un predio a la red de drenaje o cuerpo de agua superficial. El valor de vulnerabilidad con los niveles de FTIP varía desde 0.072 a 1 .

Figura 1: Modelo conceptual con los factores de transporte del Índice de Fósforo

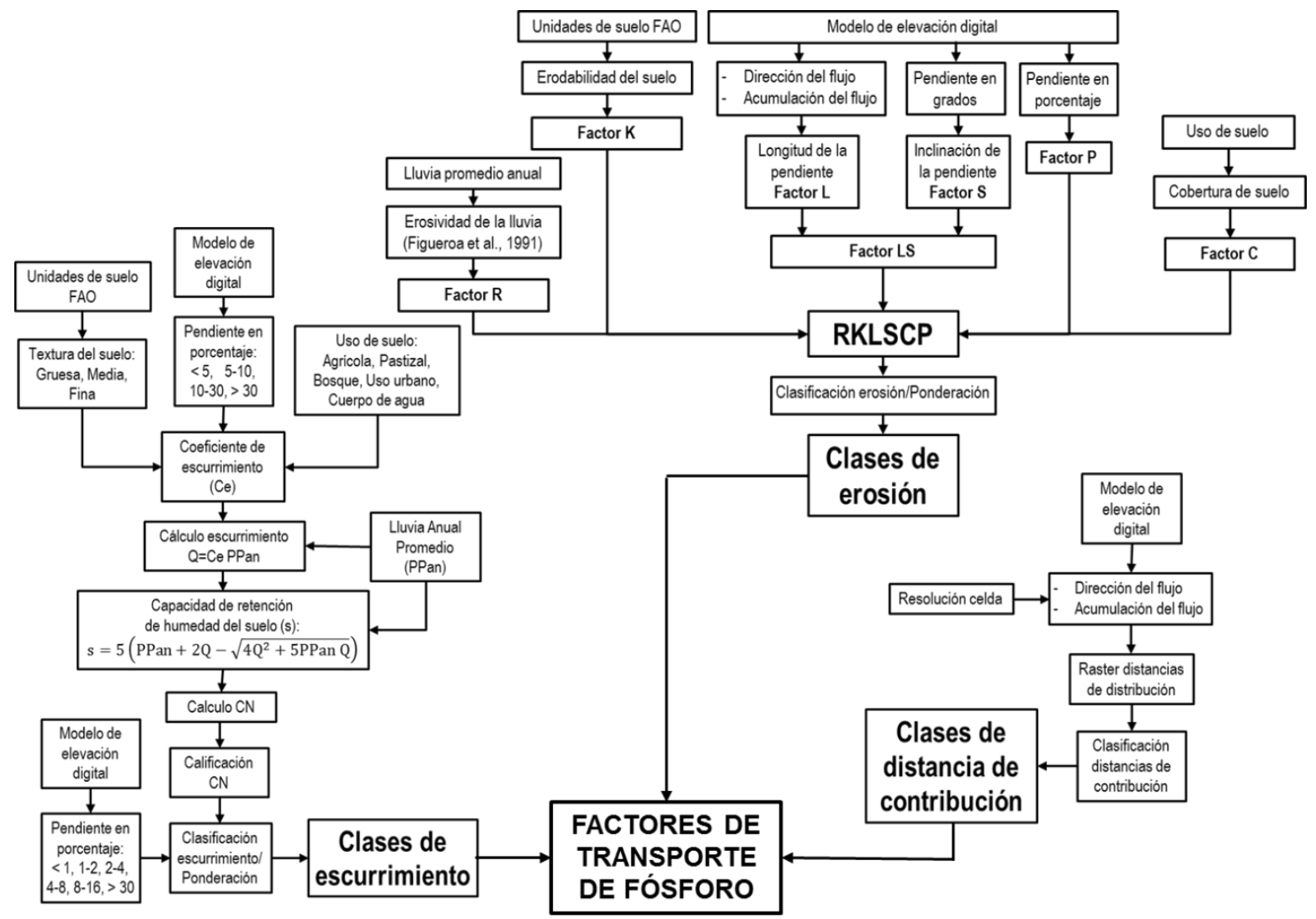




\section{Factores de transporte de fósforo}

Se constituye por la erosión hídrica, el escurrimiento superficial y el periodo de retorno o la distancia a los cuerpos de agua o red de drenaje superficial. Cada uno de estos componentes se describe a continuación.

Erosión hídrica. La erosión hídrica se estimó con la USLE, la cual fue diseñada para calcular la erosión laminar y en surcos de terrenos ${ }^{(25)}$; consiste en un modelo matemático multifactorial que integra seis procesos que intervienen en la erosión hídrica, como los que indica la expresión $^{(26)}$ : E $=$ R K L S C P

Donde:

E es la pérdida anual de suelo en $(\mathrm{t}) \cdot(\mathrm{ha} \cdot \mathrm{a})^{-1}$;

$\mathrm{R}$ es el factor de erosividad de la lluvia en $(\mathrm{MJ} \cdot \mathrm{mm}) \cdot(\mathrm{ha} \cdot \mathrm{h})^{-1}$;

$\mathrm{K}$ es el factor de erosionabilidad del suelo $\left(\mathrm{en} \mathrm{t}^{\cdot} \mathrm{ha}^{-1}\right) \cdot(\mathrm{ha} \cdot \mathrm{h}) \cdot(\mathrm{MJ} \cdot \mathrm{mm})^{-1}$;

L es el factor de longitud de la pendiente (adimensional);

$\mathrm{S}$ es el factor del grado de la pendiente (adimensional);

$\mathrm{C}$ es el factor de manejo del cultivo (adimensional);

$\mathrm{P}$ es el factor de prácticas mecánicas para el control de la erosión (adimensional).

El factor R. Se generaron mapas con la precipitación promedio anual del área de estudio para los escenarios climáticos 1961-2010, 2021-2040, 2041-2060 y 2061-2080 ${ }^{(27)}$. El factor R se estimó para cada escenario climático, utilizando las ecuaciones que presentó Figueroa et al ${ }^{(28)}$, correspondientes a las regiones IV, VII y X de la República Mexicana, en las cuales se localiza el estado de Jalisco. Los modelos aplicados fueron los siguientes: (Región IV) $\mathrm{Y} 2.8959 \mathrm{X}+0.002893 \mathrm{X}^{2}$ con $\mathrm{R}^{2}=0.92$, (Región VII) $\mathrm{Y}=0.0334 \mathrm{X}+0.006661 \mathrm{X}^{2}$ con $\mathrm{R}^{2}=$ 0.98 y (Región $\mathrm{X}) \mathrm{Y}=6.8938 \mathrm{X}+0.000442 \mathrm{X}^{2}$ con $\mathrm{R}^{2}=0.95$, donde $\mathrm{Y}$ es el valor de $\mathrm{EI}_{30}$ anual en $\mathrm{MJ} \cdot \mathrm{mm} \cdot(\mathrm{ha} \cdot \mathrm{h})^{-1}$ y X es la lluvia promedio anual en milímetros. Para la localidad de Tepatitlán de Morelos, Jalisco, localizada en la región VII, Flores ${ }^{(29)}$ estimó la erosividad de la lluvia en 2002 y 2003 con precipitación anual de 1,074.2 y 1,264.75 mm anuales, respectivamente. Este autor utilizó la ecuación correspondiente a esta región VII de Figueroa et $a l^{(28)}$ y el modelo de Wischmeier y Smith ${ }^{(26)}$ para estimar la erosividad. El modelo de la región VII tiene tendencia a incrementar la erosividad cuando aumenta la lluvia de acuerdo con los datos de precipitación disponibles en 2002 y 2003, la erosividad fue de 9,400 y 10,183 $(\mathrm{MJ} \cdot \mathrm{mm}) \cdot(\mathrm{ha} \cdot \mathrm{h})^{-1}$, respectivamente. Con la lluvia promedio anual en el periodo de $1983 \mathrm{a}$ 2017 de $890.2 \mathrm{~mm}$, la erosividad estimada con la ecuación para la región VII fue 5,255 $(\mathrm{MJ} \cdot \mathrm{mm}) \cdot(\mathrm{ha} \cdot \mathrm{h})^{-1}$. Este valor es menor al ocurrido en 2002 y 2003 , debido a que en estos años la lluvia fue por encima de la media de la localidad. 
El factor K. Se utilizaron los valores indicados por Figueroa et al ${ }^{(28)}$, para cada una de las unidades de suelo de las cartas edafológicas de INEGI $^{(30)}$, para el estado de Jalisco con la clasificación edáfica de FAO.

El factor longitud de la pendiente del suelo (L). Para calcular el factor de longitud de la pendiente (L) se utilizó la función: $\mathrm{L}=\left(\frac{\lambda}{22.13}\right)^{\mathrm{m}}$, donde $\lambda$ es la longitud de la pendiente en $\mathrm{m}, m$ es un exponente con la modificación propuesta por Foster et $a l^{(31)}: \mathrm{m}=\frac{\beta}{(1+\beta)}, \beta=$ $\frac{\left(\frac{\operatorname{sen} \theta}{0.0896}\right)}{3.0(\operatorname{sen} \theta)^{0.8}+0.56}$, donde $\theta$ es el ángulo de la pendiente en grados. La longitud de la pendiente para cada píxel se ajustó con la relación siguiente: $\lambda=\frac{90}{\cos \theta}^{(32)}$. El valor promedio de cada píxel fue de $90 \mathrm{~m}$.

El factor inclinación de la pendiente $(\mathrm{S})$. Se calculó con las ecuaciones siguientes: $\mathrm{S}=$ $10.8 \operatorname{sen} \theta+0.03$, si $S<9 \%, S=16.8 \operatorname{sen} \theta-0.50$, si $S \geq 9$, donde $\theta$ es el ángulo de la pendiente en $\operatorname{grados}^{(32)}$.

El factor de cobertura y manejo del cultivo (C). El uso del suelo procedió de los mapas vectoriales de INEGI de la serie IV. Para los terrenos con uso agrícola se consideró que estuvieran cultivados con maíz, por lo que en este uso se aplicó el factor $\mathrm{C}=0.433$; en terrenos con pastizal se adjudicó un valor de $\mathrm{C}=0.16$. Otros valores de $\mathrm{C}$ para el uso de suelo se reportan por Flores et $a l^{(33)}$.

El factor prácticas mecánicas (P). Los valores del factor P para el control de la erosión en terrenos agrícolas, se utilizaron los recomendados por Williams et al ${ }^{(34)}$. Estos son una relación entre el porcentaje de la pendiente y la longitud máxima de los surcos trazados en contorno y solo se aplicaron a los suelos con uso agrícola de temporal. En terrenos con otros usos (ganadero y forestal), el valor de P fue igual a 1, pues en ellos se supuso no se desarrollan prácticas mecánicas.

Escurrimiento superficial. El efecto del escurrimiento superficial sobre el transporte de fósforo se evaluó con base en el Número de Curva (CN, por sus siglas en inglés). El cálculo de $\mathrm{CN}$ siguió el siguiente procedimiento:

a) Se estimó el parámetro de retención de humedad (s) con el uso de volumen de escurrimiento medio y la cantidad de lluvia, con la expresión ${ }^{(35)}: \mathrm{s}=5\left(\mathrm{PP}_{a n}+2 \mathrm{Q}-\sqrt{4 \mathrm{Q}^{2}+5 \mathrm{PP}_{a n} \mathrm{Q}}\right)$, donde $\mathrm{Q}$ es la lámina de escurrimiento medio anual en mm, PPan es la lluvia promedio anual $(\mathrm{mm})$ y s es un parámetro de retención de humedad del suelo $(\mathrm{mm})$. El volumen de escurrimiento medio se estimó por medio de la expresión ${ }^{(36)}: \mathrm{Q}=\mathrm{cPP}_{a n}$, donde $\mathrm{Q}$ es la lámina de escurrimiento anual en mm, c es el coeficiente de escurrimiento superficial y PPan es la precipitación promedio anual en $\mathrm{mm}^{(1)}$. El valor de c se determinó con la información del uso 
del suelo, la pendiente y textura del suelo en el área de estudio, con valores indicados por Flores-López et $a l^{(37)}$. La PPan sirvió de base para el cálculo de $\mathrm{R}$ en los escenarios climatológicos en estudio para Jalisco. El uso del suelo procedió de los mapas vectoriales de INEGI de la serie IV; la textura se obtuvo de mapas edafológicos de la serie III de INEGI y la pendiente se obtuvo del modelo de elevación digital de INEGI.

b) El CN se determinó con base en el parámetro s, a partir de la ecuación siguiente ${ }^{(38)}$ : $\mathrm{CN}=$ $\frac{25,400}{s+254}$. Con el CN estimado, este se combina con el valor de la pendiente para determinar la clase de escurrimiento.

Distancia de un predio a la red de drenaje o cuerpo de agua superficial. La distancia del sitio de origen al punto de conexión con la red de drenaje o cuerpo de agua superficial se determinó en ARCGIS con los comandos flow direction y flow accumulation, aplicados sobre el modelo de elevación digital de INEGI para Jalisco.

\section{Escenarios de cambio climático}

Se utilizó la mediana de 11 modelos de circulación general (MCG) de precipitación mensual generados por Ruiz-Corral $e t a l^{(27)}$, pertenecientes al CMIP5 (Intercomparación de Modelos Acoplados Fase 5): BCC-CSM1-1, CCSM4, GISS-E2-R, HadGEM2-AO, HadGEM2-ES, IPSL-CM5A-LR， MIROC-ESM-CHEM， MIROC-ESM， MIROC5， MRI-CGCM3, NorESM1-M. Se utilizaron los resultados reducidos y calibrados de lluvia de dos rutas representativas de concentración de gases efecto invernadero (RCP, por sus siglas en inglés): RCP4.5 y RCP8.5, aplicadas a tres climatologías en el área de estudio: 2030, 2050 y 2070, y en la línea base de lluvia la climatología de 1961 a 2010, identificada en el análisis como 2010 y generada en estudio previo por Ruíz-Corral et $a l^{(27)}$.

\section{Análisis de la información}

Se estimó el FTIP con la lluvia anual de las climatologías 2010, 2030, 2050 y 2070, de acuerdo con la metodología descrita en imágenes raster con resolución de 3", para las rutas de concentración de gases de invernadero (RCP) 4.5 y 8.5 del estado de Jalisco. Se considera el mismo uso del suelo para los escenarios futuros. Los cambios en el FTIP se obtuvieron con las restas en las imágenes de los periodos 2010-2030, 2010-2050 y 2010-2070, cálculo realizado con IDRISI Selva. La tasa de cambio en la FTIP se evaluó con la pendiente de regresión lineal entre la superficie de estratos de FTIP en los años de evaluación para las RCP 4.5 y 8.5 . 


\section{Resultados y discusión}

\section{Factor de Transporte del Índice de Fósforo (FTIP) en el escenario RCP4.5}

En las Figuras 2a, b, c y d se muestra el FTIP de las tierras de Jalisco con RCP 4.5 en las climatologías 2010, 2030, 2050 y 2070, respectivamente. El FTIP para la climatología base varió de 0.072 a 0.54 , con la climatología de 2030 y 2050 fue 0.072 a 0.491 , para la climatología 2070 cambió de 0 a 0.486 . En el Cuadro 1 se presenta la superficie ocupada por los niveles de vulnerabilidad a la pérdida de $\mathrm{P}$.

Figura 2: Factor de transporte del Índice de Fósforo para el escenario RCP 4.5 en las climatologías: a) 2010, b) 2030, c) 2050 y d) 2070

a)

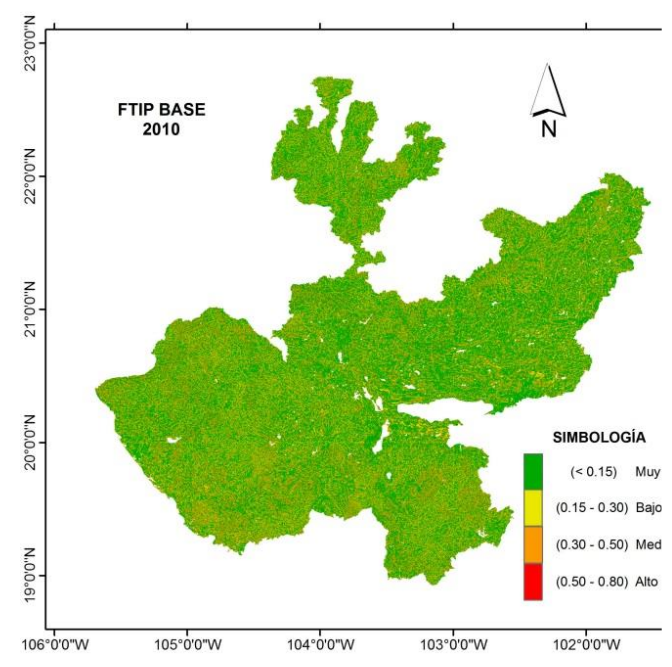

c)

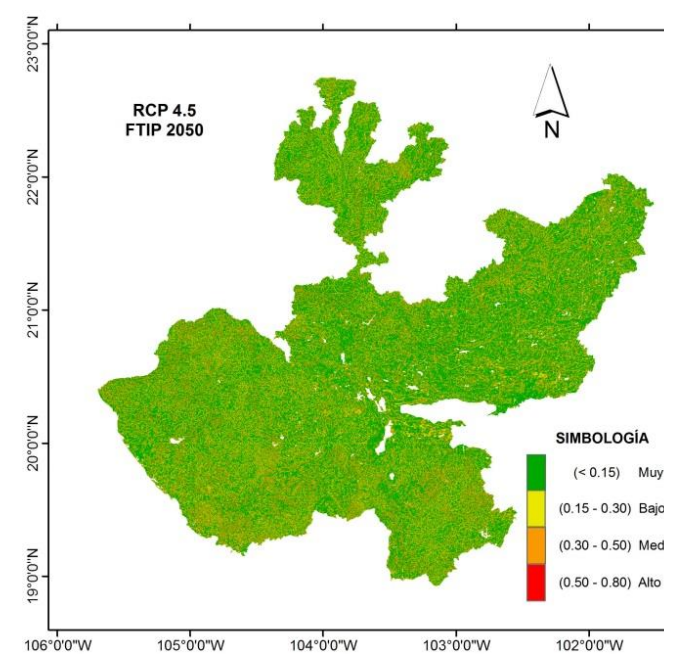

b)

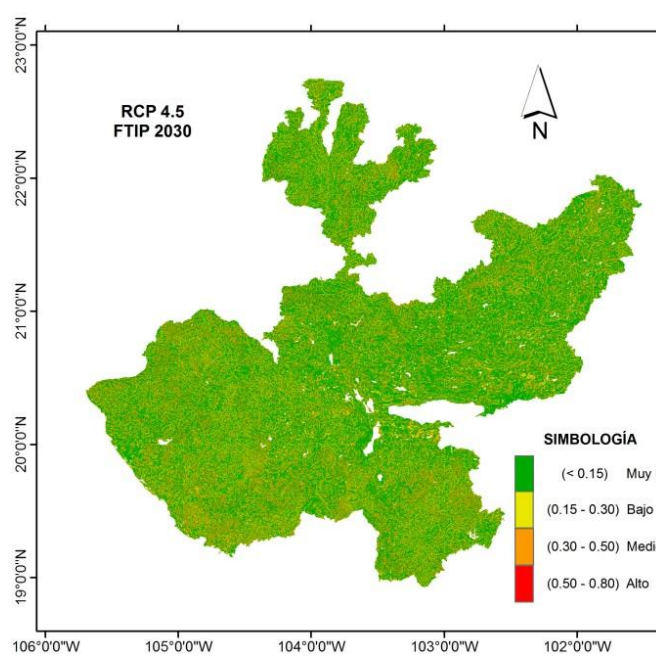

d)

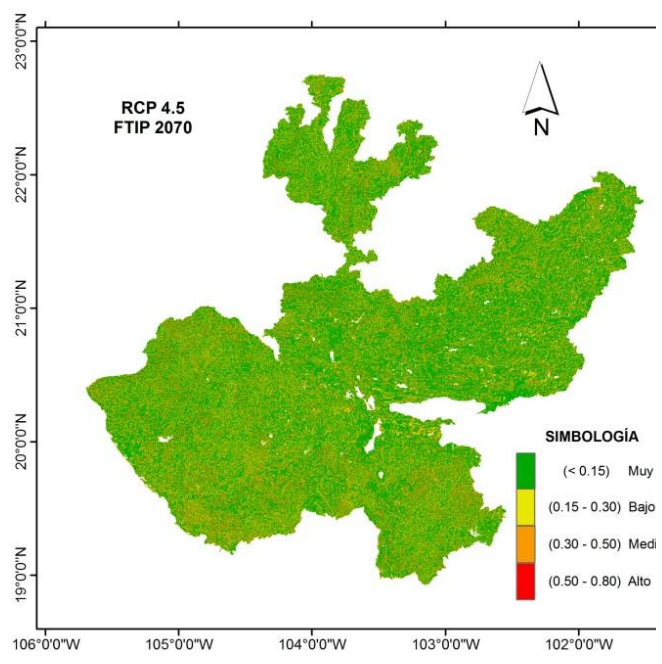


Cuadro 1: Superficie que ocupa cada estrato con el Factor de Transporte del Índice de Fósforo (FTIP) en escenario de cambio climático RCP4.5 con tres climatologías

\begin{tabular}{llllll}
\hline Nivel de vulnerabilidad & \multicolumn{4}{l}{ FTIP } & por año de climatología (miles de ha) \\
\hline Descripción & Valor FTIP & $\mathbf{2 0 1 0}$ & $\mathbf{2 0 3 0}$ & $\mathbf{2 0 5 0}$ & $\mathbf{2 0 7 0}$ \\
\hline Muy Bajo & $<0.15$ & $4,682.6$ & $4,676.9$ & $4,675.2$ & $4,674.1$ \\
Bajo & 0.15 a 0.30 & 889.3 & 906.5 & 913.7 & 919.8 \\
Medio & 0.30 a 0.50 & $2,188.3$ & $2,177.0$ & $2,171.4$ & $2,166.3$ \\
Alto & 0.50 a 0.80 & 0.109 & 0 & 0 & 0 \\
\hline
\end{tabular}

En la línea base, el nivel de vulnerabilidad a la pérdida de fósforo en las tierras se clasifica desde riesgo muy bajo a alto, mientras que, en las climatologías de 2030, 2050 y 2070, el nivel de riesgo va de muy bajo a medio y el nivel alto desaparece. El nivel de vulnerabilidad por el FTIP muy bajo $(<0.15)$ ocupa la mayor superficie, seguido del nivel medio $(0.30$ a 0.50) y bajo (0.15 a 0.30). La tendencia que tiene cada estrato del FTIP del escenario RCP4.5 en las climatologías estudiadas con la superficie ocupada, se muestra con las pendientes de los modelos de regresión lineal mostrada en el Cuadro 5. Estas pendientes muestran que las superficies en los niveles de vulnerabilidad muy bajo y medio tienen mayor tendencia a disminuir por año, mientras en el nivel bajo la tendencia es a incrementarse.

Dado que el riesgo de transporte de fósforo se asocia con la movilidad que genera el agua, produciendo desprendimiento de partículas por el salpicado del agua de lluvia y su energía cinética contenida, el flujo de agua superficial y subsuperficial ${ }^{(39)}$. Este proceso se identifica en los valores de riesgo medio y alto del escenario RCP4.5 y climatologías estudiadas, asociados principalmente a las tierras con cercanía a las redes de drenaje o cuerpos de agua. Este resultado coincide con otros estudios realizados en escala de cuenca ${ }^{(40,41,42)}$. Por esta razón, el valor de vulnerabilidad por FTIP actual con respecto al escenario RCP 4.5 en las climatologías evaluadas no refleja grandes cambios, ya que las cantidades de lluvia estimadas para Jalisco en las climatologías futuras no se esperan incrementos importantes o inclusive, en algunas áreas la lluvia se reduce provocando una disminución de riesgo del FTIP en los niveles muy bajo y medio, agregándose esta superficie en el nivel de riesgo bajo que tiende a aumentar. Esta tendencia es similar a la estimada en el FTIP para el lago Poyang de China ${ }^{(43)}$ en los escenarios de cambio climático RCP2.6, 4.5 y 8.5, aun incluyendo cambios en la intensidad de eventos extremos y su frecuencia.

La comparación del FTIP de la línea base con la estimada en las climatologías 2030, 2050 y 2070 del escenario climático RCP4.5, se muestra en el Cuadro 2. Los cambios negativos del FTIP señalan que en el escenario futuro el índice aumenta, por el contrario, cuando el cambio es positivo, el índice disminuye y la superficie de la climatología futura se reduce. En este contexto, el cambio positivo en el FTIP implica una reducción en el riesgo por contaminación 
difusa de P. El periodo 2010 a 2030 presenta la mayor superficie con un cambio negativo en el FTIP, pero en el periodo 2010-2050 y 2010-2070, la situación se invierte con mayor FTIP en 2010 con respecto a 2050 y 2070. Esto implica mayor riesgo por contaminación difusa de P en el periodo 2010-2030 y menor en las climatologías del 2010-2050 y 2010-2070 para las tierras de Jalisco.

Cuadro 2: Superficie con cambios esperados en el Factor de Transporte del Índice de Fósforo (FTIP) en las climatologías 2030, 2050 y 2070 con relación a 2010, bajo el escenario RCP4.5

\begin{tabular}{llll}
\hline \multirow{2}{*}{ Nivel de cambio en el FTIP } & \multicolumn{4}{l}{ Superficie (en miles de ha) } \\
\cline { 2 - 4 } & $\mathbf{2 0 1 0}$ a 2030 & $\mathbf{2 0 1 0}$ a 2050 & $\mathbf{2 0 1 0}$ a 2070 \\
\hline Cambio negativo $(<0)$ & $7,492.4$ & 11.5 & 18.5 \\
Cambio positivo $(\geq 0)$ & 267.9 & $7,748.7$ & $7,741.8$ \\
\hline
\end{tabular}

La precipitación pluvial de las climatologías 2030 a 2070 en el escenario del cambio climático RCP4.5, no manifestó grandes incrementos en la lluvia anual utilizada por el modelo del IP. La modificación más importante se espera en los patrones de lluvia con eventos de mayor intensidad ${ }^{(16)}$, pero el modelo de FTIP solo utiliza la lluvia anual en la climatología de la línea base y climatologías futuras. Con eventos extremos en la lluvia futura, los efectos posiblemente se reflejarán en mayor erosión hídrica y más cantidad de escurrimientos superficiales, sin embargo, el conocimiento actual no permite identificar estas características en los modelos de predicción climática ${ }^{(16,18)}$.

\section{Factor de Transporte del Índice de Fósforo (FTIP) en el escenario RCP8.5}

En las Figuras 3 a, b, c y d, se muestra la distribución del FTIP para las tierras de Jalisco en los escenarios RCP8.5 de las climatologías 2010, 2030, 2050 y 2070. A partir de estos mapas se identificaron los estratos por nivel de vulnerabilidad de FTIP mostrado en el Cuadro 3. El FTIP para la climatología base y las climatologías de 2030, 2050 y 2070 varió de 0.03 a 0.54, con generación de los estratos con FTIP mostrados en el Cuadro 3. Los estratos con la mayor superficie fueron el muy bajo (FTIP < 0.15) y medio (FTIP $0.30-0.50$ ), con tendencia a reducir el factor de transporte de las climatologías futuras, mientras en los estratos bajo y alto, no obstante que tuvieron baja superficie, su tendencia fue a incrementarse en las climatologías futuras. 
Figura 3: Factor de Transporte del Índice de Fósforo para el escenario RCP 8.5 en las climatologías: a) 2010, b) 2030, c) 2050 y d) 2070

a)

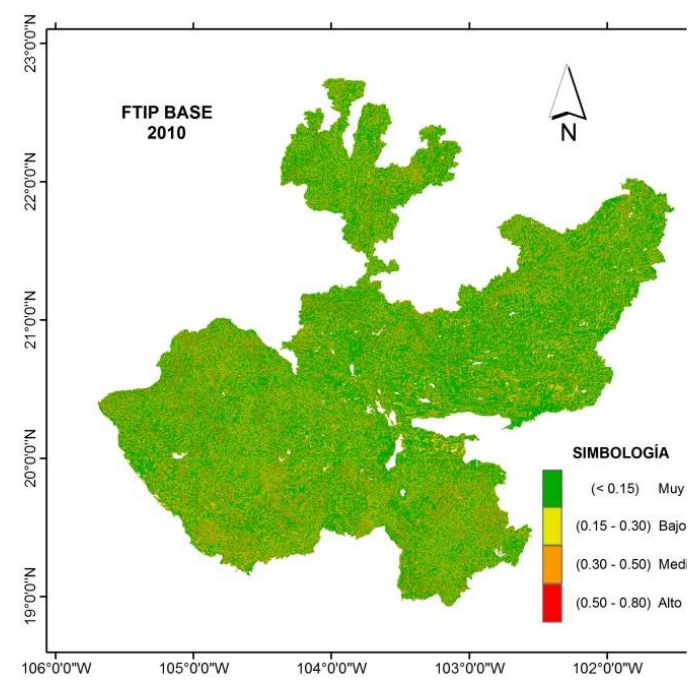

c)

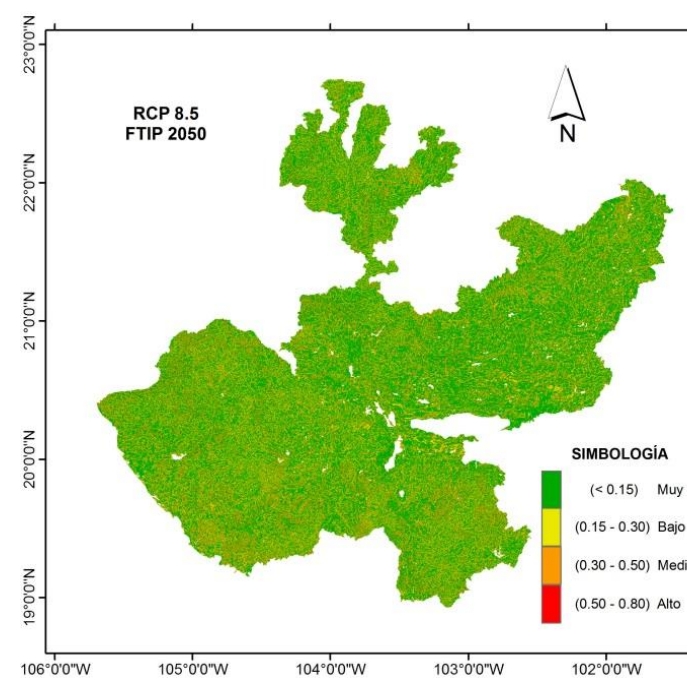

b)

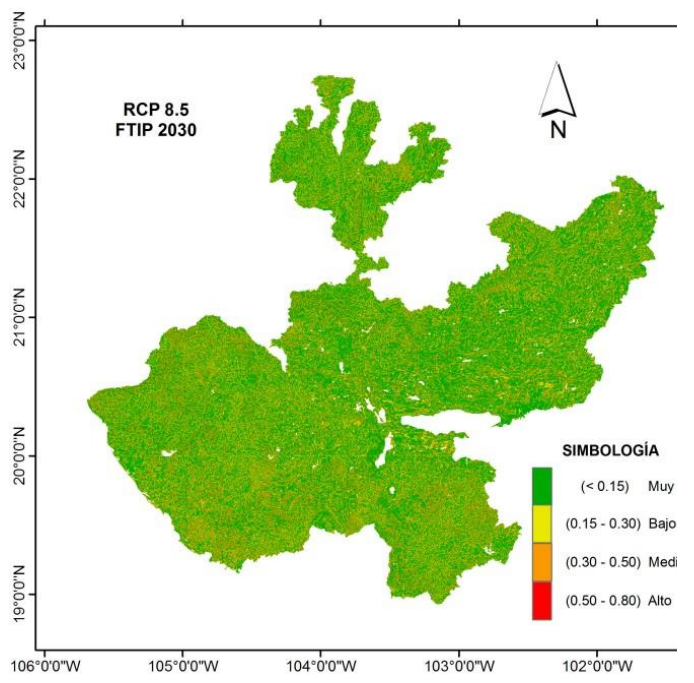

d)

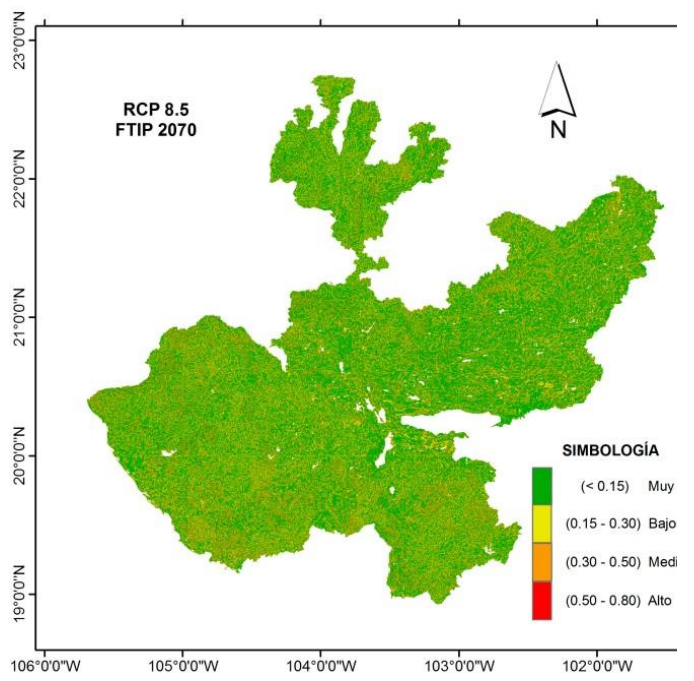

Cuadro 3: Superficie por estrato de Factor de Transporte del Índice de Fósforo (FTIP) en la climatología de referencia y tres climatologías futuras bajo el escenario RCP8.5

\begin{tabular}{llllll}
\hline \multicolumn{2}{l}{ Nivel de vulnerabilidad } & \multicolumn{4}{l}{ FTIP } \\
\hline Descripción & Valor FTIP & 2010 & 2030 & 2050 & 2070 \\
\hline Muy bajo & $<0.15$ & $4,682.6$ & $4,675.1$ & $4,673.9$ & $4,672.0$ \\
Bajo & 0.15 a 0.30 & 889.3 & 919.6 & 931.3 & 944.2 \\
Medio & 0.30 a 0.50 & $2,188.3$ & $2,165.3$ & $2,154.5$ & $2,143.3$ \\
Alto & 0.50 a 0.80 & 0.109 & 0.269 & 0.486 & 0.825 \\
\hline
\end{tabular}


La comparación del valor de FTIP en las climatologías 2010 a 2030, de 2010 a 2050 y de 2010 a 2070, se muestra en el Cuadro 4, donde se resume el cambio en las superficies asociadas con los niveles del FTIP de este escenario y climatologías estudiadas. Esta comparación produjo cambios en la superficie de FTIP desde menores a cero hasta mayores de 0.10 , todos calificados como niveles de vulnerabilidad muy bajo. En el estrato con FTIP menor a 0, la superficie fue mayor en 2030 con respecto a 2010 en más de 54 mil ha, pero en los periodos 2010-2050 y 2010-2070 este estrato desaparece.

Cuadro 4: Superficie estimada por el cambio de nivel en el Factor de Transporte del Índice de Fósforo (FTIP), al pasar de 2010 a 2030, 2050 y 2070 bajo el escenario RCP8.5

\begin{tabular}{llll}
\hline \multirow{2}{*}{ Nivel de cambio en FTIP } & \multicolumn{3}{l}{ Cambio de climatología (en miles de ha) } \\
\cline { 2 - 4 } & $\mathbf{2 0 1 0}$ a 2030 & $\mathbf{2 0 1 0}$ a 2050 & $\mathbf{2 0 1 0}$ a 2070 \\
\hline$<0$ & 54.6 & 0 & 0 \\
$0-0.05$ & $7,703.5$ & $5,242.2$ & $5,257.6$ \\
$0.05-0.10$ & 0.283 & 448.8 & 440.7 \\
$>0.10$ & 1.8 & $2,069.3$ & $2,062.0$ \\
\hline
\end{tabular}

El estrato de FTIP de 0 a 0.05 mostró la mayor superficie en el periodo 2010-2030, con reducción importante en la superficie para los periodos 2010-2050 y 2010-2070. En los estratos de 0.05-0.10 y mayor de 0.10, del FTIP se incrementa la superficie, particularmente en el estrato con FTIP mayor de 0.10 en los periodos 2010-2050 y 2010-2070. Estos cambios son atribuidos a la modificación esperada de mayor cantidad de lluvia, la cual genera un aumento en la pérdida de fósforo en las tierras agrícolas, resultado similar también reportado en el RCP 8.5 del lago Poyang de China ${ }^{(43)}$.

Las tasas de cambio observadas en las superficies de cada nivel de vulnerabilidad del FTIP y las climatologías de estudio se muestran en el Cuadro 5. Aunque la respuesta observada en el FTIP es muy baja, es producto de los mínimos cambios de la precipitación pluvial de las climatologías 2030, 2050 y 2070 del escenario RCP 8.5; también es un reflejo del pequeño aumento en la lluvia anual utilizada por el modelo del IP. Por esta razón, es posible que se esté subestimando el FTIP, ya que se espera una modificación en los patrones de lluvia con eventos de mayor intensidad ${ }^{(16,44)}$, que el modelo FTIP no considera en sus componentes de erosión del suelo y escurrimiento superficial. Al respecto, el 7 de septiembre de 2003 Flores $^{(29)}$ reporta para Tepatitlán, Jalisco, evento de lluvia de $150.05 \mathrm{~mm}$ en $24 \mathrm{~h}$ con intensidad máxima en $30 \mathrm{~min}$ de $68.5 \mathrm{~mm} / \mathrm{h}$. Una posible solución es calcular la erosión hídrica y escurrimiento superficial a escala mensual o inclusive diaria, como se indica en el FTIP $^{(11)}$, para utilizarse con la información climática futura ${ }^{(27)}$. 
Cuadro 5: Modelos de regresión lineal entre la superficie ocupada por estrato de nivel de vulnerabilidad de FTIP con el año de climatología

\begin{tabular}{llllll}
\hline $\begin{array}{l}\text { Nivel de vulnerabilidad } \\
\text { Valor }\end{array}$ & Escenario RCP 4.5 & \multicolumn{2}{l}{ Escenario RCP 8.5 } & \\
Descripción & FTIP & Modelo & $\mathbf{R}^{\mathbf{2}}$ & Modelo & $\mathbf{R}^{\mathbf{2}}$ \\
\hline Muy bajo & $<0.15$ & $\mathrm{y}=-0.136 \mathrm{x}+4953.8$ & 0.86 & $\mathrm{y}=-0.165 \mathrm{x}+5012.0$ & 0.84 \\
Bajo & 0.15 a 0.30 & $\mathrm{y}=0.495 \mathrm{x}-101.6$ & 0.94 & $\mathrm{y}=0.882 \mathrm{x}-877.7$ & 0.94 \\
Medio & 0.30 a 0.50 & $\mathrm{y}=-0.357 \mathrm{x}+2904.8$ & 0.96 & $\mathrm{y}=-0.729 \mathrm{x}+3649.8$ & 0.96 \\
Alto & 0.50 a 0.80 & & & $\mathrm{y}=0.012 \mathrm{x}-23.7$ & 0.97 \\
\hline
\end{tabular}

Los modelos de estimación de erosividad de la lluvia con escala mensual están logrando buenos resultados en estudios recientes ${ }^{(45,46)}$, así como la generación de nuevas funciones matemáticas para escalas de tiempo mensuales y diarias ${ }^{(47)}$, que para México es importante desarrollarlas dadas las predicciones de cambio climático esperadas ${ }^{(16)}$, y que a la fecha poco avance se tiene. Aunque los actuales modelos para el cálculo de erosividad de lluvia ${ }^{(28)}$ muestran la tendencia a incrementar la agresividad de la lluvia con el aumento en la lluvia anual, es recomendable evaluar este índice en condiciones más amplias a la mencionada en el presente estudio. Pero además es urgente obtener estimaciones de lluvia futura a escalas diaria, pues con la escala mensual o anual es posible se produzcan sobreestimación de tales eventos $^{(48,49)}$.

Aunque las superficies con el FTIP resultaron similares en los escenarios RCP4.5 y RCP8.5 en las climatologías estudiadas, el riesgo de contaminación difusa de $\mathrm{P}$ persiste con nivel de riesgo alto en las áreas con cercanía a los cuerpos de agua y red de drenaje superficial, sitios en los que se debe poner atención actualmente y para escenarios futuros, diseñando buenas prácticas agrícolas que permitan contener la contaminación difusa de dichas áreas.

\section{Conclusiones e implicaciones}

Los resultados del presente estudio demuestran la factibilidad de aplicación del FITP a las condiciones de Jalisco con la climatología base de 2010 y escenarios de cambio climático con climatologías futuras propuestas. Con resultados obtenidos se logró identificar las tendencias de la ruta de concentración de gases efecto invernadero bajo los escenarios RCP 4.5 y RCP 8.5 en Jalisco. Para el escenario RCP4.5, el cambio negativo en el FTIP implicó un aumento, lo que significa un incremento en el riesgo por contaminación difusa de $\mathrm{P}$, pero con un cambio positivo el riesgo por contaminación difusa de $\mathrm{P}$ se reduce. En cambio, para la RCP8.5, se identificó la mayor superficie con vulnerabilidad de muy bajo y medio, con tendencia a reducir el FTIP, mientras los estratos con nivel de vulnerabilidad bajo y alto, la tendencia fue a incrementarse. De manera general, el FTIP en los escenarios RCP 4.5 y 8.5 de las climatologías evaluadas no reflejan grandes cambios en el valor de vulnerabilidad por 
FTIP, ya que las cantidades de lluvia estimadas para Jalisco en las climatologías futuras no se esperan incrementos importantes. Dado que el modelo FTIP se calcula con lluvia anual, en esta escala de tiempo no se consideran los patrones de lluvia con eventos de alta intensidad o mayor cantidad esperado en los escenarios de cambio climático, por lo que es recomendable desarrollar funciones para estimar la erosividad de la lluvia y escurrimiento con escala mensual o inclusive diaria, en el cálculo del FTIP, en el cálculo del escurrimiento superficial y la erosión hídrica. En los escenarios RCP y climatologías estudiadas, las zonas con cercanía a los cuerpos de agua y red de drenaje superficial, representan la mayor vulnerabilidad al FTIP.

\section{Literatura citada:}

1. Zhou B, Vogt RD, Xu C, Lu X, Xu H, Bishnu JP, Zhu L. Establishment and validation of an amended phosphorus index: Refined phosphorus loss assessment of an agriculture watershed in Northern China. Water Air Soil Pollut 2014;(225):2103.

2. Sharpley AN, Daniel TC, Edwards DR. Phosphorus movement in the landscape. J Produ Agric 1993;6(4):492-500.

3. Flores LHE, Ireta MJ, Pérez DJF, Ruíz CRC, Díaz MP. Identificación de buenas prácticas agrícolas para reducir la degradación del suelo e incrementar la calidad del agua. Jalisco, México. INIFAP. 2009.

4. FAO. Food and Agriculture Organization. Reporte de la iniciativa de la ganadería, el medio ambiente y el desarrollo - Integración por zonas de la ganadería y de la agricultura especializadas (AWI) - Opciones para el manejo de efluentes de granjas porcícolas de la zona centro de México. 2003. http://www.fao.org/wairdocs/LEAD/X6372S/x6372s00.htm Consultado 28 Jun, 2007.

5. SIAP. Servicio de Información Agroalimentaria y Pesquera. Estadísticas de producción. 2016. Tomado de: http://www.gob.mx/siap/ Consultado 12 Oct, 2016.

6. De La Mora OC, Flores LHE, García VJ, Chávez DAA, Ruíz CJA. Caracterización taxonómica del plancton en la presa El Jihuite en Tepatitlán de Morelos, Jalisco. Jalisco, México: INIFAP. 2011.

7. Flores LHE, Hernández JAL, Figueroa VU, Castañeda VAA. Calidad Microbiológica del agua por contaminación difusa de la aplicación de estiércoles en maíz y pasto. Tecnologías y Ciencias del Agua. TyCA-RETAC 2012; (III):127-141.

8. Román MMR. Confort térmico y características del sistema de producción de bovinos de leche en la cuenca hidrográfica el Jihuite de los Altos de Jalisco [tesis licenciatura]. Tepatitlán de Morelos, Jalisco: Universidad de Guadalajara; 2009. 
9. Flores LHE, Figueroa VU, De La Mora OC, Núñez GG, Valdivia GL. Evaluación y calibración del índice de fósforo en los Altos de Jalisco, México. Rev Mex Cienc Agríc 2014;5(3):367-378.

10. Flores LHE, Paredes MR, Ruvalcaba GJM, De La Mora OC, Pérez DJF, Ireta MJ. Metodología para la evaluación del valor agregado del programa de maíz de alto rendimiento (PROEMAR) 2010 en Jalisco y Guanajuato. Jalisco, México. INIFAP. 2011.

11. Gburek WJ, Sharpley AN, Heathwaite L, Folmar GJ. Phosphorus management at the watershed scale: a modification of the phosphorus index. J Environ Quality 2000; (29):130-144.

12. Sharpley AN, Weld JL, Beegle DB, Kleinman PJA, Gburek WJ, Moore Jr PA, Mullins G. Development of phosphorus indices for nutrient management planning strategies in the United States. J Soil Water Conserv 2003;(58):137-152.

13. Dechmi F, Isidoro D, Stambouli T. A phosphorus index for use in intensive irrigated areas. Soil Use Manage 2013;29 (Suppl 1):64-75.

14. Marjerison RD, Dahlke H, Easton ZM, Seifert S, Walter MT. A Phosphorus Index transport factor based on variable source area hydrology for New York State. J Soil Water Conserv 2011;(66):149-157.

15. Mallarino AP, Stewart BM, Baker JL, Downing JD, Sawyer JE. Phosphorus indexing for cropland: Overview and basic concepts of the Iowa phosphorus index. J Soil Water Conserv 2002;57(6):440-447.

16. IPCC. Intergovernmental Panel on Climate Change. Climate Change 2014: Synthesis Report. Contribution of Working Groups I, II and III to the Fifth Assessment Report of the Intergovernmental Panel on Climate Change [Core Writing Team. Pachauri RK and Meyer LA, editors]. IPCC, Geneva, Switzerland. 2014.

17. Burlando P, Rosso R. Extreme storm rainfall and climatic change. Atmos Res 1991; (27):169-189.

18. Zhang GH, Nearing MA, Liu BY. Potential effects of climate change on rainfall erosivity in the yellow river basin of china. Trans ASAE 2005;(48):511-517.

19. Diodato N, Bellocchi G, Romano N, Chirico GB. How the aggressiveness of rainfalls in the Mediterranean lands is enhanced by climate change. Climatic Change 2011;(108):591-599.

20. Guhathakurta P, Sreejith OP, Menon PA. Impact of climate change on extreme rainfall events and flood risk in India. J Earth Syst Sci 2011;120(3):359-373. 
21. Heckrath G, Bechmann M, Ekholm P, Ule`n B, Djodjic F, Andersen HE. Review of indexing tools for identifying high risk area of phosphorus loss in Nordic catchments. J Hydrol 2008;(349):68-87.

22. NASEM. National Academies of Sciences, Engineering, and Medicine. Attribution of Extreme Weather Events in the Context of Climate Change. Washington, DC: The National Academies Press; 2016.

23. SIAP. Servicio de Información Agroalimentaria y Pesquera. SIACON 2017. https://www.gob.mx/siap/acciones-y-programas/produccion-agricola-33119

Consultado 15 Dic, 2017.

24. Troitiño F, Trasar-Cepeda C, Leirós MC, Gil-Sotres F. Validation and modification of the phosphorus loss index as applied to a small catchment. Soil Use Manage 2013;29(Suppl 1):114-123.

25. Wischmeier WH. Use and misuse of the universal soil loss equation. J Soil Water Conserv 1976;31(1):5-9.

26. Wischmeier WH, Smith DD. Predicting rainfall erosion losses-a guide to conservation planning. Agriculture Handbook 537. USDA, Washington, DC; 1978.

27. Ruiz-Corral JR, Medina-García G, Rodríguez-Moreno VM, Sánchez-González JJ, Villavicencio-García R, Durán Puga N, et al. Regionalización del cambio climático en México. Rev Mex Cienci Agríc 2016; Pub Esp (13):2451-2464.

28. Figueroa SB, Amante OA, Cortés THG, Pimentel LJ, Osuna CES, Rodríguez OJM, Morales FFJ. Manual de predicción de pérdidas de suelo por erosión. San Luis Potosí, México: SARH-Colegio de Posgraduados; 1991.

29. Flores LHE. Rutas de transporte superficial de nitrógeno y fósforo en un área de drenaje de Jalisco, México [tesis doctorado]. Montecillo, Texcoco, Estado DE México: Colegio de Posgraduados; 2004.

30. Instituto Nacional de Estadística, Geografía e Informática (INEGI). Cartas Edafológicas, escala 1:1’000,000. México, DF; 1993.

31. Foster GR, Meyer LD, Onstad CA. A runoff erosivity factor and variable slope length exponents for soil loss estimates. Trans ASAE 1977;(20):683-687.

32. McCool DK, Brown LC, Foster GR, Mutchler CK, Meyer CK. Revised slope steepness factor for the Universal Soil Loss Equation. Trans ASAE 1987;10(5):1387-1396.

33. Flores LHE, Pérez DJF, Ireta MJ. Estimación de la erosión hídrica en agave tequilero en Jalisco. Jalisco, México. INIFAP; 2010. 
34. Williams JR, Dyke PT, Fuchs WW, Benson VW, Rice OW, Taylor ED. EPICErosion/Productivity Impact Calculator: 2 User manual. Technical Bulletin 1768. USDA-ARS, Temple, Texas. 1990.

35. Jasso IR, Sánchez CI, Stone JJ, Melgoza CA, Simanton JR, Martínez RJG. Estimación de parámetros para la modelación del escurrimiento superficial y erosión hídrica. En: Sánchez CI, et al, editores. Uso de la lluvia artificial para parametrizar modelos de procesos hidrológicos. Gómez Palacio, Durango. INIFAP. 1999.

36. SARH-CP. Manual de Conservación del Suelo y del Agua. 2da ed. Texcoco, México: Colegio de Postgraduados; 1982.

37. Flores LHE, Martínez MM, Oropeza MJL, Mejía SE, Carrillo GR. Integración de la EUPS a un SIG para estimar la erosión hídrica del suelo en una cuenca hidrográfica de Tepatitlán, Jalisco, México. Terra 2003;(21):233-244.

38. NRCS. Natural Resources Conservation Service. Phosphorus Assessment Tool for Texas. USDA-NRCS. Texas, USA; 2012.

39. Schoumans OF, Chardon W. Risk assessment methodologies for predicting phosphorus losses. J Plant Nutr Soil Sc 2003;(166):403-408.

40. Reichmann O, Chen Y, Iggy LM. Spatial model assessment of $P$ transport from soils to waterways in an Eastern Mediterranean watershed. Water 2013;(5):262-279.

41. Zhou H, Gao C. Assessing the risk of phosphorus loss and identifying critical source areas in the Chaohu Lake Watershed, China. Environ Manage 2011;(48):1033-1043.

42. Ortega-Achury SL, Martinez-Rodriguez GA, Sotomayor-Ramirez DR, Ramirez-Avila JJ. Caribbean phosphorus index validation and management practices evaluation on fields under manure applications. An ASABE Meeting Presentation Paper. Reno, Nevada. 2013.

43. Jiang S, Zhang Q. Modelling phosphorus transport and its response to climate change at upper stream of Poyang Lake-the largest fresh water lake in China. Geophys Res Abst 2017;(19):EGU2017-2365-1.

44. Meelh GA, Arblaster JM, Tebaldi C. Understanding future patterns of increased precipitation intensity in climate model simulations. Geophys Res Lett 2005;(32):L18719.

45. Lee MH, Lin HH. Evaluation of annual rainfall erosivity index based on daily, monthly, and annual precipitation data of rainfall station network in southern Taiwan. INT J DISTRIB SENS N 2015; http://journals.sagepub.com/doi/full/10.1155/2015/214708 Accessed 14 Dec, 2017. 
46. Sadeghi SH, Tavangar S. Development of stational models for estimation of rainfall erosivity factor in different timescales. Nat Hazards 2015;77(1):429-443 https://link.springer.com/article/10.1007/s11069-015-1608-y Accessed 14 Dec, 2017.

47. Bonilla CA, Vidal KL. 2011. Rainfall erosivity in Central Chile. J Hydrol 2011;(410):126-133.

48. Sun Y, Solomon S, Dai A, Portmann RW. How often does it rain? J climate 2006; (19):916-934.

49. Sun Y, Solomon S, Dai A, Portmann RW. How often will it rain? J climate 2007; (20):4801-4818. 